\title{
Advances in Receptor-Targeted Radiolabeled Peptides for Melanoma Imaging and Therapy
}

\author{
Yubin Miao ${ }^{1}$ and Thomas P. Quinn ${ }^{2}$ \\ ${ }^{1}$ Department of Radiology, School of Medicine, University of Colorado Denver, Aurora, Colorado; and ${ }^{2}$ Department of Biochemistry, \\ University of Missouri-Columbia, and Harry S. Truman Veterans' Hospital, Columbia, Missouri
}

\begin{abstract}
Melanocortin-1 receptor (MC1R) and very late antigen-4 (VLA-4, integrin $\alpha_{4} \beta_{1}$ ) are 2 attractive molecular targets for developing peptide radiopharmaceuticals for melanoma imaging and therapy. MC1R- and VLA-4-targeting peptides and peptide-conjugated Cornell prime dots ( $\mathrm{C}^{\prime}$ dots) can serve as delivery vehicles to target both diagnostic and therapeutic radionuclides to melanoma cells for imaging and therapy. This review highlights the advances of MC1R- and VLA-4-targeted radiolabeled peptides and peptide-conjugated $\mathrm{C}^{\prime}$ dots for melanoma imaging and therapy. The promising preclinical and clinical results of these new peptide radiopharmaceuticals present an optimistic outlook for clinical translation into receptor-targeting melanoma imaging and radionuclide therapy in the future.
\end{abstract}

Key Words: melanocortin-1 receptor; very late antigen-4; peptide radiopharmaceuticals; Cornell prime dots; melanoma imaging and therapy

J Nucl Med 2021; 62:313-318

DOI: 10.2967/jnumed.120.243840

\section{$\mathbf{M}$}

alignant melanoma is the most lethal form of skin cancer, with approximately 100,350 new cases and 6,850 fatalities in the United States in 2020 (1). Metastatic melanoma is extremely aggressive, leading to the high mortality rate of melanoma. The traditional median overall survival of metastatic melanoma patients is less than 9 mo. New molecular treatments, such as vemurafenib (BRAF inhibitor), ipilimumab (targeting cytotoxic T-lymphocyte-associated protein 4), and nivolumab (inhibitor of programmed cell death protein 1), have improved the overall survival of metastatic melanoma patients by months. However, the treatments are still far from satisfactory, because the 5-y survival is approximately $35 \%$ for metastatic melanoma patients (2). Clearly, there is a great need to develop new theranostic approaches for metastatic melanoma.

Received Sep. 29, 2020; revision accepted Nov. 20, 2020.

For correspondence or reprints contact: Yubin Miao, Department of Radiology, School of Medicine, University of Colorado Denver, $12700 \mathrm{E}$. 19th Ave., Aurora, CO 80045.

E-mail: yubin.miao@cuanschutz.edu

Published online Dec. 4, 2020.

COPYRIGHT (c) 2021 by the Society of Nuclear Medicine and Molecular Imaging.
Melanin and melanocortin-1 receptor (MC1R) are 2 attractive molecular targets for melanoma. Melanin is a negatively charged dark pigment that is produced by melanocytes and exists in most melanomas and neoplastic melanocytes that ultimately develop to melanoma. MC1R is a G-protein-coupled receptor that is overexpressed on mouse and human melanoma cells $(3,4)$ and on greater than $80 \%$ of melanotic and amelanotic human metastatic melanoma samples (4). The reports of a melanin-targeting $N$-(2-(diethylamino)ethyl) ${ }^{18} \mathrm{~F}-5$-fluoropicolinamide and a MC1R-targeting ${ }^{68} \mathrm{Ga}-$ DOTA-GGNle-CycMSH ${ }_{\text {hex }}$ on melanoma patients $(5,6)$ have demonstrated the clinical relevance of both targets for melanoma imaging. Over the past several years, very late antigen-4 (VLA-4, integrin $\alpha_{4} \beta_{1}$ ) has emerged as another attractive molecular target for melanoma because of its expression on melanoma and the correlation between the expression of VLA-4 integrin and melanoma progression and metastasis (7-9).

This review focuses on the preclinical and clinical studies of melanoma using MC1R- and VLA-4-targeted radiolabeled peptides and Cornell prime dots $\left(\mathrm{C}^{\prime}\right.$ dots) to highlight the advances predominately within the past $5 \mathrm{y}$ due to its concise feature. Because it takes more than a decade to develop peptides, optimize their pharmacokinetic properties, and reach the milestone of clinical translation, several research and review articles published in the last decade are included to present a historic perspective. The promising preclinical and clinical results of new MC1Rand VLA-4-targeted peptide radiopharmaceuticals highlighted in this review present an optimistic outlook for clinical translation into receptor-targeting melanoma imaging and radionuclide therapy in the future.

\section{MC1R-TARGETED DIAGNOSTIC AND THERAPEUTIC a-MELANOCYTE-STIMULATING HORMONE (a-MSH) PEPTIDES}

MC1R has been an attractive molecular target for developing peptide radiopharmaceuticals for melanoma imaging and targeted radionuclide therapy. MC1R-targeted melanoma imaging using a radiolabeled cyclic $\alpha$-MSH peptide is illustrated in Figure 1. The $\alpha$-MSH peptide can target diagnostic radionuclides to melanoma cells for imaging and deliver therapeutic radionuclides to melanoma cells for radionuclide 


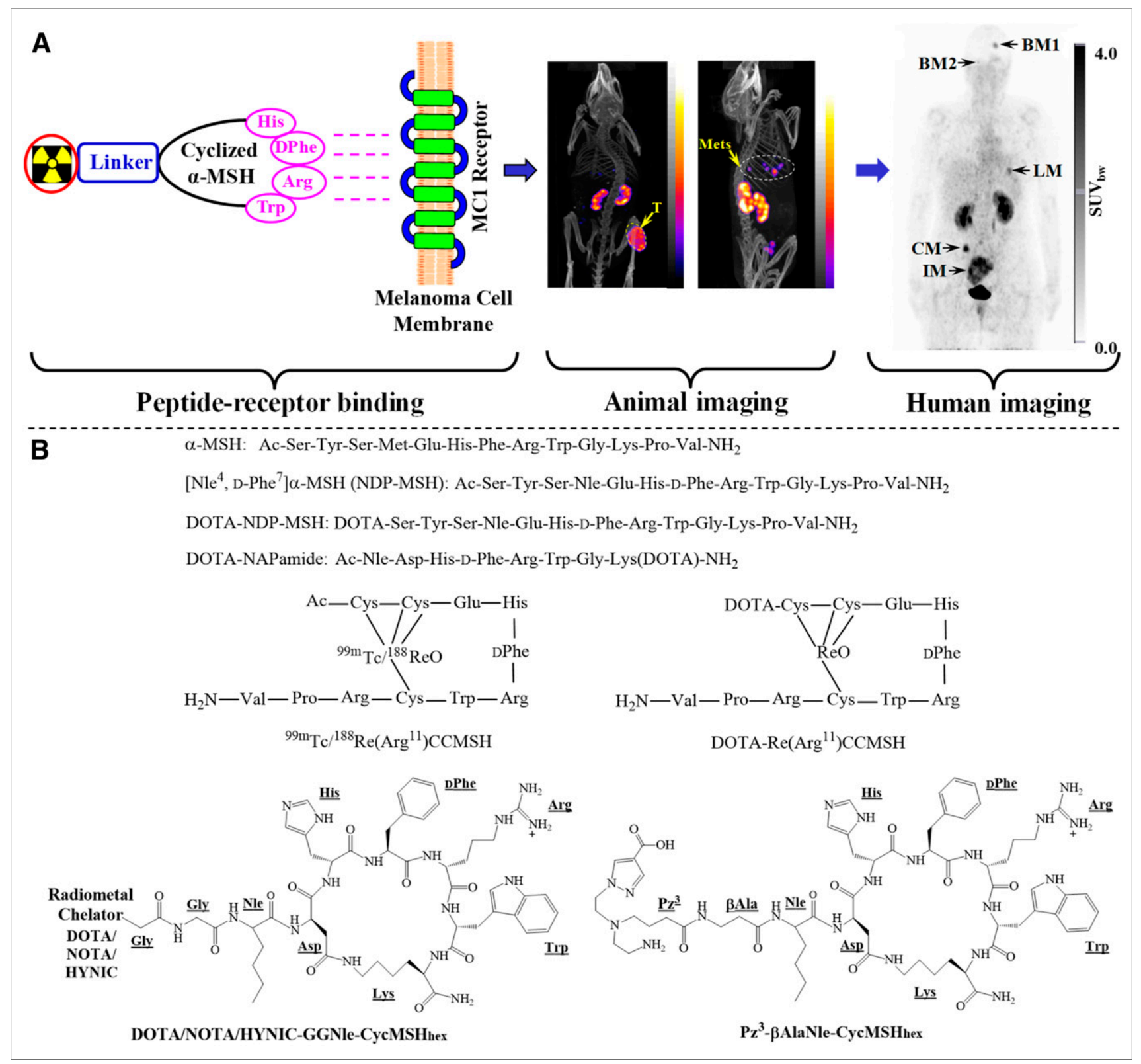

FIGURE 1. (A) Schematic illustration of MC1R-avid radiolabeled a-MSH peptide to detect B16/F1 flank melanoma (T) and B16/ F10 pulmonary metastatic melanoma (Mets) in mice and melanoma metastases in a melanoma patient. BM, LM, CM, and IM represent melanoma metastases in brain, lung, connective tissue, and intestines, respectively. (B) Representative a-MSH peptides. (Human and animal images reprinted with permission of 5,19 , and 20.)

therapy. Native $\alpha-\mathrm{MSH}$ is a linear peptide with 13 amino acids (Ac-Ser ${ }^{1}-\mathrm{Tyr}^{2}-\mathrm{Ser}^{3}-\mathrm{Met}^{4}-\mathrm{Glu}^{5}-\mathrm{His}^{6}-\mathrm{Phe}^{7}-\mathrm{Arg}^{8}-\mathrm{Trp}^{9}-\mathrm{Gly}^{10}{ }^{1} \mathrm{Lys}^{11}{ }^{11}$ $\mathrm{Pro}^{12}-\mathrm{Val}^{13} \mathrm{NH}_{2}$ ). The moiety of $\mathrm{His}^{6}-\mathrm{Phe}^{7}-\mathrm{Arg}^{8}-\mathrm{Trp}^{9}$ is an MC1R-binding sequence. Since native $\alpha-M S H$ is subject to proteolytic degradation in vivo, nonnatural amino acids such as norleucine ${ }^{4}\left(\mathrm{Nle}^{4}\right)$ and D-Phe ${ }^{7}$ were introduced to stabilize the bioactive conformation of the peptide, resulting in the discovery of NDP-MSH with subnanomolar MC1R binding affinity (10). Building on the construct of NDP-MSH, the Quinn, Eberle, and Cheng groups have reported several radiolabeled linear DOTA-NDP-MSH and DOTA-NAPamide (AcNle-Asp-His-D-Phe-Arg-Trp-Gly-Lys(DOTA)- $\mathrm{NH}_{2}$ ) peptides for melanoma imaging, and their promising preclinical results have been summarized by several review articles (11-14).

Peptide cyclization with disulfide bonding, lactam, or site-specific metal has been successfully used to enhance MC1R binding and the biologic activity of cyclic $\alpha$-MSH peptides. As compared with linear peptides, the cyclic $\alpha$-MSH peptides possess less conformational flexibility, making them better fit the MC1R-binding pocket for improved binding affinities. As shown in Figure 1, Quinn's group pioneered and developed a novel class of radiolabeled metal-cyclized $\alpha$-MSH peptides that achieved cyclization and radiolabeling simultaneously when labeled with ${ }^{99 \mathrm{~m}} \mathrm{Tc} /{ }^{188} \mathrm{Re}$. 
Building on the success of ${ }^{99 \mathrm{~m}} \mathrm{Tc}-\left(\mathrm{Arg}^{11}\right) \mathrm{CCMSH}\left(\mathrm{Cys}^{3}-\right.$ Cys ${ }^{4}-$ Glu $^{5}$-His ${ }^{6}$-D-Phe ${ }^{7}-$ Arg $^{8}-$ Trp $^{9}$-Gly ${ }^{10}$-Arg ${ }^{11}$-Pro $^{12}-$ Val $^{13}-\mathrm{NH}_{2}$ ), nonradioactive rhenium was used to cyclize the peptide when DOTA was coupled to the peptide for radiolabeling of other theranostic radionuclides, including ${ }^{111} \mathrm{In},{ }^{64} \mathrm{Cu}$, ${ }^{86} \mathrm{Y},{ }^{177} \mathrm{Lu},{ }^{90} \mathrm{Y}$, and ${ }^{212} \mathrm{~Pb}(15-17)$. Those metal-cyclized $\alpha$-MSH peptides have demonstrated promising preclinical results for melanoma imaging and therapy and have been highlighted by several review articles (11-14).

Lactam-cyclized $\alpha$-MSH peptides have been developed by the Santos and Miao groups for radiolabeling of diagnostic radionuclides $\left({ }^{99 \mathrm{~m}} \mathrm{Tc},{ }^{111} \mathrm{In},{ }^{67} \mathrm{Ga},{ }^{64} \mathrm{Cu}\right)$ for melanoma imaging over the past few years (18-22). Santos' group examined the effect of different azolyl-ring substitution patterns of pyrazolyldiamine bifunctional chelators on the pharmacokinetics of ${ }^{99 m} \mathrm{Tc}(\mathrm{CO})_{3}-\mathrm{Pz}^{1-4}-\beta$ AlaNle-CycMSH${ }_{\text {hex }} \quad$ (c[Asp-His-D-Phe-ArgTrp-Lys]-CONH 2 ) (18). ${ }^{99 m} \mathrm{Tc}(\mathrm{CO})_{3}-\mathrm{Pz}^{3}-\beta$ AlaNle-CycMSH $\mathrm{CHx}_{\text {hex }}$ exhibited high B16/F1 melanoma uptake (11.82 \pm 3.91 percentage injected dose $[\% \mathrm{ID}] / \mathrm{g}$ at $1 \mathrm{~h}$ after injection). The introduction of a carboxylate group in the azolyl ring resulted in a remarkable reduction of kidney and liver accumulation.

Miao's group used hydrazinonicotinamide (HYNIC)/ DOTA/NOTA for radiolabeling of ${ }^{99 \mathrm{~m}} \mathrm{Tc},{ }^{111} \mathrm{In},{ }^{67} \mathrm{Ga}$, and ${ }^{64} \mathrm{Cu}$, building on the construct of GlyGlyNle-CycMSH $\mathrm{MSx}_{\text {hex }}$ (19-22). The B16/F1 melanoma uptake of these radiolabeled

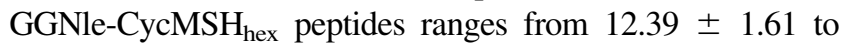
$25.53 \pm 2.22 \% \mathrm{ID} / \mathrm{g}$ at $2 \mathrm{~h}$ after injection. Interestingly, the GlyGly linker led to the favorable tumor targeting and urinary clearance for ${ }^{111} \mathrm{In}-,{ }^{67} \mathrm{Ga}-,{ }^{64} \mathrm{Cu}$-labeled DOTA/NOTA-GGNle$\mathrm{CycMSH}_{\text {hex }}$ (19-21), whereas the 8-aminooctanoic acid (Aoc) linker resulted in increased tumor uptake of ${ }^{99 \mathrm{~m}} \mathrm{Tc}$ (ethylenediamine diacetic acid)-HYNIC-AocNle-CycMSH $\mathrm{MS}_{\text {hex }}$ as compared with ${ }^{99 \mathrm{~m} T c(e t h y l e n e d i a m i n e}$ diacetic acid)-HYNIC-GGNle$\mathrm{CycMSH}_{\text {hex }}$ (22). To take advantage of the readiness of the $\left[{ }^{99 \mathrm{~m}} \mathrm{Tc}(\mathrm{CO})_{3}\left(\mathrm{OH}_{2}\right)_{3}\right]^{+}$tricarbonyl kit, they further developed ${ }^{99} \mathrm{~m} \mathrm{Tc}(\mathrm{CO})_{3}-\mathrm{NOTA}-\mathrm{GGNle}-\mathrm{CycMSH}_{\text {hex }}$ (23). Interestingly, the switch from HYNIC to NOTA dramatically increased the melanoma uptake $(19.76 \pm 3.62 \% \mathrm{ID} / \mathrm{g}$ at $2 \mathrm{~h}$ after injection) and decreased the renal and liver uptake of ${ }^{99 \mathrm{~m}} \mathrm{Tc}(\mathrm{CO})_{3}-\mathrm{NOTA}^{-G G N l e-C y c M S H} \mathrm{H}_{\text {hex }}(1.59 \pm 0.52$ and $1.57 \pm 0.32 \% \mathrm{ID} / \mathrm{g}$, respectively, at $2 \mathrm{~h}$ after injection). Importantly, NOTA-GGNle-CycMSH $\mathrm{Hex}_{\text {ex }}$ could serve as a versatile peptide platform for radiolabeling of ${ }^{99 m} \mathrm{Tc},{ }^{67 / 68} \mathrm{Ga}$, and ${ }^{64} \mathrm{Cu}$ for SPECT and PET imaging of melanoma.

Building on the success of GGNle-CycMSH $\mathrm{Hex}_{\text {ex }}$, Bénard's group replaced the GlyGly linker with 4-amino-(1-carboxymethyl) piperidine (Pip) and identified ${ }^{68}$ Ga-DOTA-Pip-Nle$\mathrm{CycMSH}_{\text {hex }}$ as a promising conjugate for further evaluation because of its high B16/F10 melanoma uptake $(21.9 \pm 4.6 \%$ ID/g at $2 \mathrm{~h}$ after injection) (24). Furthermore, they used the ammoniomethyl-trifluoroborate $\left(\mathrm{AmBF}_{3}\right)$ moiety to generate ${ }^{18} \mathrm{~F}-\mathrm{AmBF}_{3}$-Pip-Nle-CycMSH $\mathrm{CH}_{\text {hex }}$ for melanoma imaging. High B16/F10 melanoma uptake $(11.96 \pm 2.31 \% \mathrm{ID} / \mathrm{g}$ at $2 \mathrm{~h}$ after injection) and fast urinary clearance of ${ }^{18} \mathrm{~F}$ $\mathrm{AmBF}_{3}$-Pip-Nle-CycMSH $\mathrm{MSx}_{\text {hex }}$ warranted its further evaluation (25).
The persistent translation efforts with HYNIC/DOTA/ NOTA-GGNle-CycMSH $\mathrm{Mex}_{\text {hex }}$ peptides eventually led to the first-in-human study of ${ }^{68} \mathrm{Ga}$-DOTA-GGNle-CycMSH $\mathrm{MHx}_{\text {hex }}$ on melanoma patients with metastases $(5) .{ }^{68} \mathrm{Ga}$ is an attractive PET radionuclide that can readily be obtained through a commercial ${ }^{68} \mathrm{Ge} /{ }^{68} \mathrm{Ga}$ generator. Miao's and Kratochwil's groups demonstrated the MC1R specificity of ${ }^{68} \mathrm{Ga}$-DOTA-GGNle-CycMSH $\mathrm{Hex}_{\text {h }}$ on B16/F10 murine melanoma and M21 xenografted human melanoma $(24.27 \pm 3.74$ and $6.07 \pm 0.68 \% \mathrm{ID} / \mathrm{g}$, respectively, at $1 \mathrm{~h}$ after injection). Then they performed the first-in-human imaging of ${ }^{68} \mathrm{Ga}$-DOTA-GGNle-CycMSH $\mathrm{MHx}_{\text {hex }}$ on 2 melanoma patients with metastases. As shown in Figure 1, the melanoma metastases in brain, lung, connective tissue, and bulky metastases in the small intestine were clearly visualized

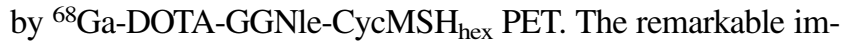
ages of those metastases in patients demonstrated the clinical relevance of MC1R as a valid and attractive molecular target for melanoma imaging, highlighted the potential of ${ }^{68}$ Ga-DOTA-GGNle-CycMSH $\mathrm{H}_{\text {hex }}$ as an MC1R-targeting probe for human melanoma imaging, and underscored the need for developing MC1R-targeting therapeutic peptides to treat metastatic melanoma patients.

The substitution of diagnostic radionuclides with therapeutic radionuclides ( $\beta$ - and $\alpha$-emitters) can generate therapeutic $\alpha$-MSH peptides for melanoma treatment. Miao's group developed and evaluated ${ }^{177} \mathrm{Lu}$ - and ${ }^{90}$ Y-DOTA-GGNle$\mathrm{CycMSH}_{\text {hex }}$ on B16/F1 and B16/F10 melanoma-bearing mice $(26,27)$. Both ${ }^{177} \mathrm{Lu}$ and ${ }^{90}$ Y-DOTA-GGNle-CycMSH ${ }_{\text {hex }}$ exhibited high MC1R-mediated melanoma uptake $(21.63 \pm 6.27$ and $19.93 \pm 5.73 \% \mathrm{ID} / \mathrm{g}$, respectively, at $2 \mathrm{~h}$ after injection) and rapid urinary clearance, warranting further evaluation for melanoma therapy. ${ }^{212} \mathrm{~Pb}$ is an attractive radionuclide for targeted $\alpha$-therapy and can easily be obtained from a ${ }^{224} \mathrm{Ra}-{ }^{212} \mathrm{~Pb}$ generator. ${ }^{212} \mathrm{~Pb}$ decays to ${ }^{212} \mathrm{Bi}$ via a $\beta$-decay $(0.57 \mathrm{MeV})$, and then ${ }^{212} \mathrm{Bi}$ eventually decays to stable ${ }^{208} \mathrm{~Pb}$ through a branched decay scheme yielding $2 \beta$-particles (1.8 and $2.2 \mathrm{MeV}$ ) and $2 \alpha$-particles (6.1 and $8.8 \mathrm{MeV})$. ${ }^{203} \mathrm{~Pb} /{ }^{212} \mathrm{~Pb}$ are attractive matched-pair theranostic radionuclides. The radiolabeling of ${ }^{203} \mathrm{~Pb} /{ }^{212} \mathrm{~Pb}$ can be achieved under identical conditions. Miao's group reported that ${ }^{203} \mathrm{~Pb}$-DOTA-GGNle-CycMSH $\mathrm{H}_{\text {hex }}$ displayed similar MC1Rspecific uptake on B16/F1 and B16/F10 melanoma lesions $(12.61 \pm 2.28$ vs. $16.81 \pm 5.48 \% \mathrm{ID} / \mathrm{g}$, respectively, at $2 \mathrm{~h}$ after injection) (28). The B16/F10 pulmonary metastatic melanoma lesions could be clearly imaged by ${ }^{203} \mathrm{~Pb}$-DOTAGGNle-CycMSH $\mathrm{MH}_{\text {hex }}$ SPECT. The favorable melanomatargeting property of ${ }^{203} \mathrm{~Pb}$-DOTA-GGNle-CycMSH $\mathrm{H}_{\text {hex }}$ warranted the evaluation of ${ }^{212} \mathrm{~Pb}$-DOTA-GGNle-CycMSH $\mathrm{H}_{\text {hex }}$ for melanoma therapy.

\section{MC1R-TARGETED a-MSH-FUNCTIONALIZED DIAGNOSTIC AND THERAPEUTIC C' DOTS}

Ultrasmall ( $<8 \mathrm{~nm}$ in diameter) hybrid inorganic core and organic shell nanomaterials are under development for MC1R-targeted multiplexed-image-guided surgery, 
radioimaging, and targeted therapy (29). $C^{\prime}$ dots are nearinfrared fluorescent silica nanoparticles that are surfacefunctionalized with polyethylene glycol (PEG) and have the ability to display biomarker targeting ligands (30). Cyclic Arg-Gly-Asp-Tyr-PEG-Cy5-C' dots have achieved investigational-new-drug approval and are in phase 1 and 2 trials for radioimaging and image-guided surgery (31).

MC1R-avid $C^{\prime}$ dots were investigated for their potential as melanoma multimodal optical (32) and radioimaging agents (29) and as targeted therapeutics $(33,34)$. PEG-Cy5$\mathrm{C}^{\prime}$ dots were surface-functionalized with the rhenium-cyclized $\alpha$-MSH peptide Ac-Cys-(Ahx) $)_{2}$-D-Lys-Re[Cys ${ }^{3}-\mathrm{Cys}^{4}-\mathrm{Glu}^{5}-$ His $^{6}$-D-Phe ${ }^{7}-\mathrm{Arg}^{8}-\mathrm{Trp}^{9}-\mathrm{Cys}^{10}{ }^{10}-\mathrm{Arg}^{11}-\mathrm{Pro}^{12}-\mathrm{Val}^{13}-\mathrm{NH}_{2}$ (Fig. 2). Multidentate peptide display yielded particles with a halfmaximal inhibitory concentration of $0.66 \mathrm{nM}$, an affinity about 10 times than that of the targeting DOTA- $\alpha$-MSH peptide. In vitro cell uptake and competitive binding studies performed with $\alpha-\mathrm{MSH}-\mathrm{C}^{\prime}$ dots demonstrated melanoma-selective cell binding, internalization, and low efflux (29).

Biodistribution studies were performed with ${ }^{125}$ I-labeled $\alpha$-MSH-PEG-Cy5-C' dots on both M21 human melanoma xenografts and B16/F10 syngenetic mouse models (29). Tissue uptake in the individual mouse models varied slightly, but the trends were similar. Favorable pharmacokinetic properties were observed, with blood levels at approximately $15-20 \% \mathrm{ID} / \mathrm{g}$ at $4 \mathrm{~h}, 5-10 \% \mathrm{ID} / \mathrm{g}$ at $24 \mathrm{~h}$, and 1 $\%$ ID/g at $72 \mathrm{~h}$. Renal clearance was more than $100 \% \mathrm{ID} / \mathrm{g}$ at $1 \mathrm{~h}$ and more than $30 \% \mathrm{ID} / \mathrm{g}$ at $4 \mathrm{~h}$. Liver and spleen uptake was low, at less than $5 \% \mathrm{ID} / \mathrm{g}$ at $24 \mathrm{~h}$. Maximal tumor uptake, 5-6 \% ID/g, was observed at $24 \mathrm{~h}$ after injection. PET imaging studies were performed with ${ }^{89} \mathrm{Zr}$ [desferrioxamine]-radiolabeled $\alpha$-MSH-PEG-Cy5-C' dots. ${ }^{89} \mathrm{Zr}$ [desferrioxamine]- $\alpha$-MSH-PEG-Cy5-C' dot PET images showed high tumor-to-background ratios at 24, 48, and $96 \mathrm{~h}$ after injection that could be blocked by coinjection

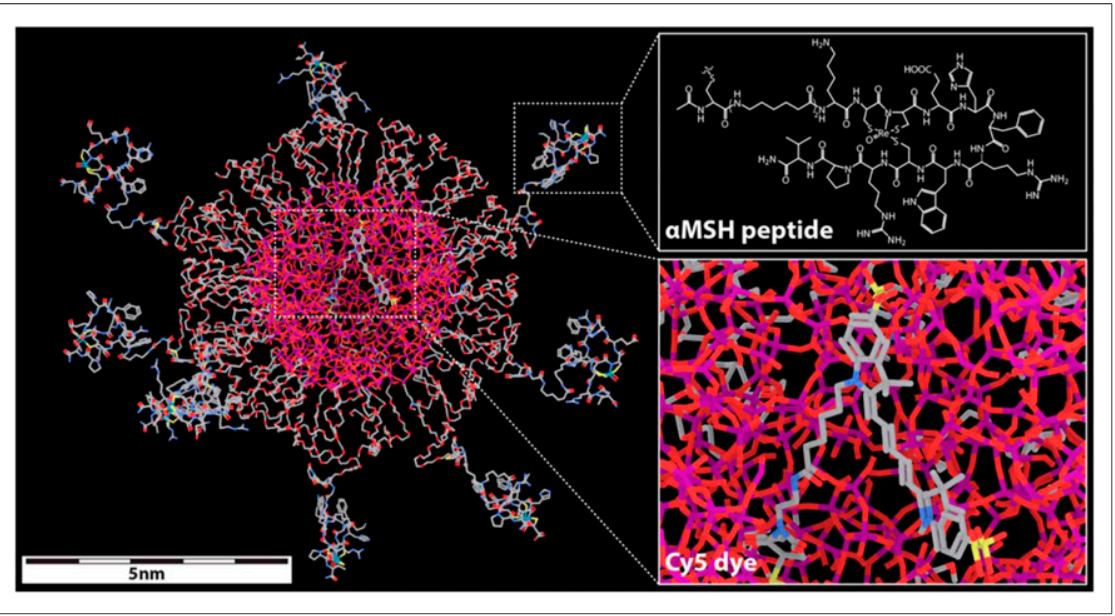

FIGURE 2. Molecular model of a-MSH-PEG-Cy5-C' dot. Silicon, oxygen, carbon, nitrogen, and sulfur atoms are colored purple, red, gray, blue, and yellow, respectively. DOTA chelator was attached to epsilon amine of D-Lys residue for ${ }^{177} \mathrm{Lu}$ and ${ }^{225} \mathrm{Ac}$ radiolabeling. (Reprinted with permission of 29.) of the NDP-MSH peptide, demonstrating tumor selectivity. Quantitation of the PET images revealed tumor uptake of $5.5 \pm 0.9 \% \mathrm{ID} / \mathrm{g}$ and liver uptake of less than $3 \% \mathrm{ID} / \mathrm{g}$.

The therapeutic potential of the DOTA- $\alpha$-MSH-PEGCy5- $C^{\prime}$ dots was examined with the $\beta$-emitter ${ }^{177} \mathrm{Lu}$ and the $\alpha$-emitter ${ }^{225} \mathrm{Ac}(33,34)$. The DOTA- $\alpha$-MSH-PEG-Cy5$\mathrm{C}^{\prime}$ dots were radiolabeled with ${ }^{177} \mathrm{Lu}$ at more than $95 \%$ radiochemical yield, resulting in a specific activity of $2.035 \times$ $10^{11} \mathrm{MBq} / \mathrm{mol}$ (33). Therapy studies were performed on M21 and B16/F10 tumor-bearing mice treated with $18.5-\mathrm{MBq}{ }^{177} \mathrm{Lu}-$ DOTA- $\alpha$-MSH-PEG-Cy5-C' dots, $18.5-\mathrm{MBq}^{177} \mathrm{Lu}-\mathrm{DOTA}-$ PEG-Cy5-C' dots, DOTA- $\alpha-M S H-P E G-C y 5-C^{\prime}$ dots, or phosphate-buffered saline vehicle control. Statistical analysis of the data demonstrated that mice receiving radiolabeled $\mathrm{C}^{\prime}$ dots had significantly better survival than those receiving nonradiolabeled $\mathrm{C}^{\prime}$ dots or phosphate-buffered saline. Importantly, there was a significant increase in survival of mice treated with $18.5-\mathrm{MBq}{ }^{177} \mathrm{Lu}-\mathrm{DOTA}-\alpha-\mathrm{MSH}-\mathrm{PEG}-\mathrm{Cy} 5-\mathrm{C}^{\prime}$ dots compared with nontargeted $18.5-\mathrm{MBq}{ }^{177} \mathrm{Lu}-\mathrm{DOTA}-$ PEG-Cy5-C' dots, demonstrating the utility of the MC1R targeting.

Therapy studies with $\alpha$-particle-emitting ${ }^{225}$ Ac-DOTA$\alpha$-MSH-PEG-Cy5-C' dots were performed on B16/F10 melanoma tumor-bearing mice (34). A 2-step approach was used to produce ${ }^{225}$ Ac-DOTA- $\alpha$-MSH-PEG-Cy5-C' dots, yielding a specific activity of $2.361 \times 10^{8} \mathrm{MBq} / \mathrm{mol}$. The therapy study included groups treated with $0.0111-\mathrm{MBq}$ ${ }^{225}$ Ac-DOTA- $\alpha$-MSH-PEG-Cy5-C' dots, 0.0111-MBq ${ }^{225} \mathrm{Ac}-$ DOTA-PEG-Cy5-C' dots, and a human serum albumin vehicle control. Kaplin-Meier analysis revealed median survival times of 26, 21, and $14 \mathrm{~d}$ after injection, respectively. A logrank test showed that the treatment groups had a statistical improvement in mean survival time and that mice treated with ${ }^{225}$ Ac-DOTA- $\alpha$-MSH-PEG-Cy5-C' dots had a statistically significant increase in survival time over ${ }^{225}$ Ac-DOTA-PEG-

Cy5-C' dot-treated mice. An analysis of macrophage, T-cell, and natural killer cell populations present in the $\alpha$-irradiated tumor microenvironment revealed dynamic and time-dependent changes, suggesting that combination immunotherapeutic approaches may increase treatment efficacy. Interestingly, the nonradioactive DOTA-PEG-Cy5-C' dots also induced comparable changes in the tumor microenvironment, although their cytotoxicity was much less than that of the ${ }^{225}$ Ac-DOTA-PEG-Cy5-C' dots.

\section{VLA-4-TARGETED DIAGNOSTIC AND THERAPEUTIC LLP2A PEPTIDES}

VLA-4 is a transmembrane noncovalent heterodimer that is widely expressed on a variety of tumors, such as melanoma, lymphoma, and multiple myeloma. 
The increased expression of the VLA-4 integrin correlates with tumor progression and development of human melanoma metastasis $(7,8)$. Lam's group used a 1-bead-1-compound library to identify a high-affinity peptidomimetic ligand, $N$-[[4-[[[(2-ethylphenyl)amino]carbonyl]amino]phenyl]acetyl]$N^{\varepsilon}$-6-[(2E)-1-oxo-3-(3-pyridinyl-2-propenyl)]-L-lysyl-L-2aminohexanedioyl-(1-amino-1-cyclohexane)carboxamide (LLP2A; half-maximal inhibitory concentration, $2 \mathrm{pM}$ ), to target VLA-4 (9). Building on the construct of LLP2A, DeNardo's group developed and evaluated ${ }^{111}$ In-labeled DOTA-conjugated LLP2A peptides with or without PEG linkers on a Raji lymphoma mouse model. They identified non-PEGylated ${ }^{111}$ In-DOTA-LLP2A as a lead VLA-4-targeting peptide for further evaluation, because of its high tumor-to-nontumor uptake ratio (35).

Anderson's group conjugated CB-TE1A1P $(1,4,8,11$ tetraazacyclotetradecane-1-(methane phosphonic acid)-8(methane carboxylic acid) to LLP2A and evaluated the melanoma-targeting property of ${ }^{64} \mathrm{Cu}-\mathrm{CB}-\mathrm{TE} 1 \mathrm{~A} 1 \mathrm{P}-\mathrm{LLP} 2 \mathrm{~A}$ on B16/F10 melanoma-bearing C57 mice (36). Despite higher uptake in VLA-4-rich spleen and bone marrow, the B16/F10 melanoma uptake for ${ }^{64} \mathrm{Cu}-\mathrm{CB}-\mathrm{TE} 1 \mathrm{~A} 1 \mathrm{P}-$ LLP2A was $11.4 \pm 2.3 \% \mathrm{ID} / \mathrm{g}$ at $2 \mathrm{~h}$ after injection. They further conjugated 2-(4,7-bis(carboxymethyl)-1,4,7-triazonan1-yl)pentanedioic acid (NODAGA) to LLP2A via a PEG $_{4}$ linker because NODAGA can form stable complexes with both ${ }^{64} \mathrm{Cu}$ and ${ }^{68} \mathrm{Ga}$ (37). The introduction of the $\mathrm{PEG}_{4}$ linker improved the binding affinity of the CBTE1A1P-LLP2A conjugate by 5 -fold. Interestingly, ${ }^{64} \mathrm{Cu}-\mathrm{CB}-$

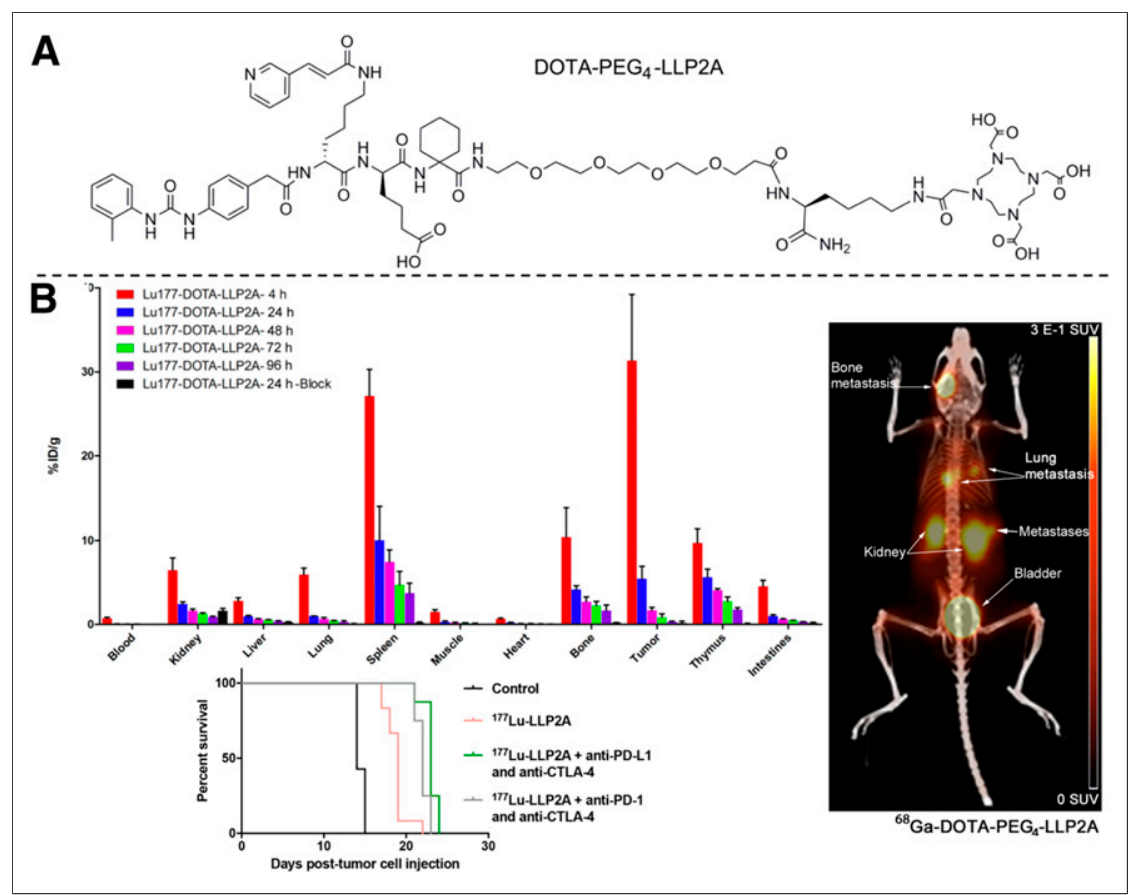

FIGURE 3. (A) Schematic structure of DOTA-PEG 4 -LLP2A. (B) Biodistribution of ${ }^{177} \mathrm{Lu} /{ }^{68} \mathrm{Ga}-\mathrm{DOTA}-\mathrm{PEG}_{4}$-LLP2A and survival curves for treatments with ${ }^{177} \mathrm{Lu}$-DOTA$\mathrm{PEG}_{4}$-LLP2A alone and combined with immune checkpoint inhibitors. (Reprinted with permission of 38 and 39.)
TE1A1P-PEG 4 -LLP2A exhibited higher tumor uptake than ${ }^{64} \mathrm{Cu}-\mathrm{NODAGA}-\mathrm{PEG}_{4}-\mathrm{LLP} 2 \mathrm{~A}(16.9 \pm 2.2$ vs. $13.4 \pm 1.7$ $\% \mathrm{ID} / \mathrm{g}$, respectively, at $4 \mathrm{~h}$ after injection), as well as better tumor-to-nontumor tissue ratios. Meanwhile, ${ }^{68} \mathrm{Ga}-\mathrm{NODAGA}-$ PEG $_{4}$-LLP2A exhibited lower B16/F10 melanoma uptake than did ${ }^{64} \mathrm{Cu}-\mathrm{CB}-\mathrm{TE} 1 \mathrm{~A} 1 \mathrm{P}-\mathrm{PEG}_{4}-\mathrm{LLP} 2 \mathrm{~A}$. However, melanoma metastases in lung, bone, and ovary could be clearly visualized by ${ }^{64} \mathrm{Cu}-\mathrm{CB}-\mathrm{TE} 1 \mathrm{~A} 1 \mathrm{P}-\mathrm{PEG}_{4}-\mathrm{LLP} 2 \mathrm{~A}$ and ${ }^{68} \mathrm{Ga}-$ NODAGA-PEG 4 -LLP2A (37).

${ }^{177} \mathrm{Lu}$ - and ${ }^{68} \mathrm{Ga}-\mathrm{DOTA}-\mathrm{PEG}_{4}$-LLP2A were prepared and evaluated on B16/F10 melanoma-bearing mice to demonstrate the potential of detecting and treating melanoma (Fig. 3) (38). ${ }^{177} \mathrm{Lu}-\mathrm{DOTA}-\mathrm{PEG}_{4}$-LLP2A showed melanoma uptake that was very high $(31.3 \pm 7.8 \% \mathrm{ID} / \mathrm{g})$ at $4 \mathrm{~h}$ and then significantly decreased (to $5.4 \pm 1.5 \% \mathrm{ID} / \mathrm{g}$ ) at $24 \mathrm{~h}$, although ${ }^{177} \mathrm{Lu}$-DOTA-PEG 4 -LLP2A exhibited relatively high uptake in VLA-4-rich spleen, thymus, and bone marrow. Meanwhile, ${ }^{68} \mathrm{Ga}-\mathrm{DOTA}-\mathrm{PEG}_{4}-\mathrm{LLP} 2 \mathrm{~A}$ displayed $9.1 \pm 0.9 \% \mathrm{ID} / \mathrm{g}$ in $\mathrm{B} 16 / \mathrm{F} 10$ tumor at $1 \mathrm{~h}$ after injection. Anderson's group then examined the therapeutic efficacy of ${ }^{177}$ Lu-DOTA-PEG 4 -LLP2A, alone and combined with immune checkpoint inhibitors (anti-programmed cell death protein 1 plus anti-cytotoxic T-lymphocyte-associated protein 4 , and anti-programmed cell death ligand 1 plus anticytotoxic T-lymphocyte-associated protein 4), on B16/F10 melanoma-bearing mice (39). ${ }^{177}$ Lu-DOTA-PEG 4 -LLP2A treatment (29.97 MBq/mouse) alone exhibited therapeutic efficacy and prolonged the median survival time of the control group from 14 to $19 \mathrm{~d}$, whereas the combination of ${ }^{177} \mathrm{Lu}-$ DOTA-PEG 4 -LLP2A and immune checkpoint inhibitors significantly enhanced the median survival time to $22-23 \mathrm{~d}$ (Fig. 3) (39). The promising therapeutic results warranted further preclinical studies to optimize the combination of therapeutic regimens.

Building on the promising results of radiometal-labeled LLP2A derivatives, Bénard's group replaced $\mathrm{PEG}_{4}$ linker with $\mathrm{PEG}_{2}$ linker and used ${ }^{18} \mathrm{~F}-\mathrm{AmBF}_{3}$ to yield DOTA- $\left({ }^{18} \mathrm{~F}-\mathrm{AmBF}_{3}\right)-\mathrm{PEG}_{2}-\mathrm{LL}$ $\mathrm{P} 2 \mathrm{~A}$ (40). Interestingly, the introduction of DOTA as a hydrophilic moiety increased the B16/F10 tumor uptake of DOTA- $\left({ }^{18} \mathrm{~F}-\mathrm{AmBF}_{3}\right)-\mathrm{PEG}_{2}$-LLP2A to $9.46 \pm 2.19 \% \mathrm{ID} / \mathrm{g}$ and reduced its gastrointestinal accumulation to $4.55 \pm 0.80$ $\% \mathrm{ID} / \mathrm{g}$ at $1 \mathrm{~h}$ after injection as compared with ${ }^{18} \mathrm{~F}-\mathrm{AmBF}_{3}-\mathrm{PEG}_{2}-\mathrm{LLP} 2 \mathrm{~A}$. However, high uptake in VLA-4-positive spleen and bone marrow (28.33 \pm 4.28 and $8.23 \pm 0.84 \% \mathrm{ID} / \mathrm{g}$ at $1 \mathrm{~h}$ after injection) may indicate that these will become dose-limiting organs when the VLA-4-targeted therapeutic applications are pursued in future studies. 


\section{CONCLUSION}

Receptor-targeting peptide radiopharmaceuticals continue to receive great interest toward the development of theranostic agents for melanoma. The new MC1R- and VLA-4-targeted radiolabeled peptides and peptide-conjugated $\mathrm{C}^{\prime}$ dots highlighted here represent major advances that will likely have an impact on translational imaging and radionuclide therapy for melanoma. The promising preclinical and clinical results of these new peptide radiopharmaceuticals present an optimistic outlook for clinical translation into receptor-targeting melanoma imaging and radionuclide therapy in the future.

\section{DISCLOSURE}

Financial support was received from NIH R01CA225837, U54 CA199081-01, and P30 CA00874CCSG. No other potential conflict of interest relevant to this article was reported.

\section{ACKNOWLEDGMENTS}

We thank the University of Missouri Peptide Synthesis Core and the Harry Truman Veterans' Hospital Biomolecular Imaging Center and Pharmacology Core for their support and the MSKCCCornell Center for Translation of Cancer Nanomedicines.

\section{REFERENCES}

1. Siegel RL, Miller KD, Jemal A. Cancer statistics, 2020. CA Cancer J Clin. 2020;70:7-30

2. Weiss SA, Wolchok JD, Sznol M. Immunotherapy of melanoma: facts and hopes. Clin Cancer Res. 2019;25:5191-5201.

3. Siegrist W, Solca F, Stutz S, et al. Characterization of receptors for alphamelanocyte-stimulating hormone on human melanoma cells. Cancer Res. 1989; 49:6352-6358.

4. Tatro JB, Wen Z, Entwistle ML, et al. Interaction on an $\alpha$-melanocyte stimulating hormone-diphtheria toxin fusion protein with melanotropin receptors in human metastases. Cancer Res. 1992;52:2545-2548.

5. Yang J, Xu J, Gonzalez R, Lindner T, Kratochwil C, Miao Y. ${ }^{68}$ Ga-DOTAGGNle-CycMSH $_{\text {hex }}$ targets the melanocortin-1 receptor for melanoma imaging. Sci Transl Med. 2018;10:eaau4445.

6. Ma X, Wang S, Wang S, et al. Biodistribution, radiation dosimetry, and clinical application of a melanin-targeted PET probe, ${ }^{18} \mathrm{~F}-\mathrm{P} 3 \mathrm{BZA}$, in patients. $\mathrm{J} \mathrm{Nucl}$ Med. 2019;60:16-22.

7. Schadendorf D, Heidel J, Gawlik C, Suter L, Czarnetzki BM. Association with clinical outcome of expression of VLA-4 in primary cutaneous malignant melanoma as well as P-selectin and E-selectin on intratumoral vessels. J Natl Cancer Inst. 1995;87:366-371.

8. Kuphal S, Bauer R, Bosserhoff AK. Integrin signaling in malignant melanoma. Cancer Metastasis Rev. 2005;24:195-222.

9. Peng L, Liu R, Marik J, Wang X, Takada Y, Lam KS. Combinatorial chemistry identifies high-affinity peptidomimetics against $\alpha_{4} \beta_{1}$ integrin for in vivo tumor imaging. Nat Chem Biol. 2006;2:381-389.

10. Sawyer TK, Sanfilippo PJ, Hruby VJ, et al. 4-norleucine, 7-D-phenylalaninealpha-melanocyte-stimulating hormone: a highly potent alpha-melanotropin with ultralong biological activity. Proc Natl Acad Sci USA. 1980;77:5754-5758.

11. Miao Y, Quinn TP. Alpha-melanocyte stimulating hormone peptide-targeted melanoma imaging. Front Biosci. 2007;12:4514-4524.

12. Eberle AN, Rout B, Qi MB, Bigliardi PL. Synthetic peptide drugs for targeting skin cancer: malignant melanoma and melanotic lesions. Curr Med Chem. 2017;24:1797-1826.

13. Miao Y, Quinn TP. Peptide-targeted radionuclide therapy for melanoma. Crit Rev Oncol Hematol. 2008;67:213-228.

14. Quinn T, Zhang X, Miao Y. Targeted melanoma imaging and therapy with radiolabeled alpha-melanocyte stimulating hormone peptide analogues. G Ital Dermatol Venereol. 2010;145:245-258.

15. Miao Y, Benwell K, Quinn TP. ${ }^{99 \mathrm{~m}} \mathrm{Tc}-$ and ${ }^{111}$ In-labeled $\alpha$-melanocytestimulating hormone peptides as imaging probes for primary and pulmonary metastatic melanoma detection. J Nucl Med. 2007;48:73-80.
16. McQuade P, Miao Y, Yoo J, Quinn TP, Welch MJ, Lewis JS. Imaging of melanoma using ${ }^{64} \mathrm{Cu}$ and ${ }^{86} \mathrm{Y}$-DOTA-ReCCMSH$\left(\mathrm{Arg}^{11}\right)$, a cyclized peptide analogue of $\alpha$-MSH. J Med Chem. 2005;48:2985-2992.

17. Miao Y, Hylarides M, Fisher DR, et al. Melanoma therapy via peptide-targeted $\alpha$-radiation. Clin Cancer Res. 2005;11:5616-5621.

18. Morais M, Oliveira BL, Correia JD, et al. Influence of the bifunctional chelator on the pharmacokinetic properties of ${ }^{99 \mathrm{~m}} \mathrm{Tc}(\mathrm{CO})_{3}$-labeled cyclic $\alpha$-melanocyte stimulating hormone analog. J Med Chem. 2013;56:1961-1973.

19. Guo H, Yang J, Gallazzi F, Miao Y. Effects of the amino acid linkers on the melanoma-targeting and pharmacokinetic properties of ${ }^{111}$ In-labeled lactam bridge-cyclized $\alpha$-MSH peptides. J Nucl Med. 2011;52:608-616.

20. Guo H, Gallazzi F, Miao Y. Ga-67-labeled lactam bridge-cyclized alpha-MSH peptides with enhanced melanoma uptake and reduced renal uptake. Bioconjug Chem. 2012;23:1341-1348.

21. Guo H, Miao Y. Cu-64-labeled lactam bridge-cyclized alpha-MSH peptides for PET imaging of melanoma. Mol Pharm. 2012;9:2322-2330.

22. Guo H, Miao Y. Introduction of an aminooctanoic acid linker enhances uptake of Tc-99m-labeled lactam bridge-cyclized alpha-MSH peptide in melanoma. $\mathrm{J} \mathrm{Nucl}$ Med. 2014;55:2057-2063.

23. Qiao Z, Xu J, Gonzalez R, Miao Y. Novel [ $\left.{ }^{99 \mathrm{~m}} \mathrm{Tc}\right]$-tricarbonyl-NOTA-conjugated lactam-cyclized alpha-MSH peptide with enhanced melanoma uptake and reduced renal uptake. Mol Pharm. 2020;17:3581-3588.

24. Zhang C, Zhang Z, Lin KS, et al. Preclinical melanoma imaging with ${ }^{68} \mathrm{Ga}$-labeled $\alpha$-melanocyte-stimulating hormone derivatives using PET. Theranostics. 2017;7:805-813.

25. Zhang $\mathrm{C}$, Zhang Z, Lin KS, et al. Melanoma imaging using ${ }^{18}$ F-labeled $\alpha$-melanocyte-stimulating hormone derivatives with positron emission tomography. Mol Pharm. 2018;15:2116-2122

26. Guo H, Miao Y. Melanoma targeting property of a Lu-177-labeled lactam bridgecyclized alpha-MSH peptide. Bioorg Med Chem Lett. 2013;23:2319-2323.

27. Xu J, Yang J, Gonzalez R, Fisher DR, Miao Y. Melanoma-targeting property of Y-90-labeled lactam-cyclized alpha-melanocyte-stimulating hormone peptide. Cancer Biother Radiopharm. 2019;34:597-603.

28. Yang J, Xu J, Cheuy L, Gonzalez R, Fisher DR, Miao Y. Evaluation of a novel $\mathrm{Pb}$-203-labeled lactam-cyclized alpha-melanocyte-stimulating hormone peptide for melanoma targeting. Mol Pharm. 2019;16:1694-1702.

29. Chen F, Zhang X, Ma K, et al. Melanocortin-1 receptor-targeting ultrasmall silica nanoparticles for dual-modality human melanoma imaging. ACS Appl Mater Interfaces. 2018;10:4379-4393.

30. Ma K, Mendoza C, Hanson M, Werner-Zwanziger U, Zwanziger J, Weisener U. Control of ultrasmall sub-10 nm ligand-functionalized fluorescent core-shell silica nanoparticle growth in water. Chem Mater. 2015;27:4119-4133.

31. Phillips E, Penate-Medina O, Zanzonico PB, et al. Clinical translation of an ultrasmall inorganic optical-PET imaging nanoparticle probe. Sci Transl Med. 2014;6:260ra149.

32. Chen F, Madajewski B, Ma K, et al. Molecular phenotyping and image-guided surgical treatment of melanoma using spectrally distinct ultrasmall core-shell silica nanoparticles. Sci Adv. 2019;5:eaax5208.

33. Zhang X, Chen F, Turker MZ, et al. Targeted melanoma radiotherapy using ultrasmall ${ }^{177} \mathrm{Lu}$-labeled $\alpha$-melanocyte stimulating hormone-functionalized core-shell silica nanoparticles. Biomaterials. 2020;241:119858.

34. Urbanska AM, Khanin R, Alidori S, et al. A genomic profile of local immunity in the melanoma microenvironment following treatment with $\alpha$ particle-emitting ultrasmall silica nanoparticles. Cancer Biother Radiopharm. 2020;35:459-473.

35. DeNardo SJ, Liu R, Albrecht $\mathrm{H}$, et al. ${ }^{111} \mathrm{In}-\mathrm{LLP} 2 \mathrm{~A}-\mathrm{DOTA}$ polyethylene glycol-targeting $\alpha_{4} \beta_{1}$ integrin: comparative pharmacokinetics for imaging and therapy of lymphoid malignancies. J Nucl Med. 2009;50:625-634.

36. Jiang M, Ferdani R, Shokeen M, Anderson CJ. Comparison of two cross-bridged macrocyclic chelators for the evaluation of ${ }^{64} \mathrm{Cu}$-labeled-LLP2A, a peptidomimetic ligand targeting VLA-4-positive tumors. Nucl Med Biol. 2013;40:245-251.

37. Beaino W, Anderson CJ. PET imaging of very late antigen-4 in melanoma: comparison of ${ }^{68} \mathrm{Ga}$ - and ${ }^{64} \mathrm{Cu}$-labeled NODAGA and CB-TE1A1P-LLP2A conjugates. J Nucl Med. 2014;55:1856-1863.

38. Beaino W, Nedrow JR, Anderson CJ. Evaluation of ${ }^{68} \mathrm{Ga}$ - and ${ }^{177} \mathrm{Lu}-\mathrm{DOTA}-$ PEG $_{4}$-LLP2A for VLA-4-targeted PET imaging and treatment of metastatic melanoma. Mol Pharm. 2015;12:1929-1938.

39. Choi J, Beaino W, Fecek RJ, et al. Combined VLA-4-targeted radionuclide therapy and immunotherapy in a mouse model of melanoma. J Nucl Med. 2018; 59:1843-1849

40. Roxin Á, Zhang C, Huh S, et al. A metal-free DOTA-conjugated ${ }^{18}$ F-labeled radiotracer: $\left[{ }^{18} \mathrm{~F}\right] \mathrm{DOTA}-\mathrm{AMBF}_{3}-\mathrm{LLP} 2 \mathrm{~A}$ for imaging VLA-4 over-expression in murine melanoma with improved tumor uptake and greatly enhanced renal clearance. Bioconjug Chem. 2019;30:1210-1219. 